\title{
Particle Spectra from the ALCOR Model
}

\author{
J. Zimányi!n, T.S. Biró, T. Csörgö, P. Lévai \\ Research Institute for Particle and Nuclear Physics, \\ H-1525 Budapest, 114. POB 49, Hungary
}

\begin{abstract}
We introduce the Transchemistry Model as a dynamical extension of the Algebraic Coalescence Rehadronization (ALCOR) model in describing the hadronization of quark matter which is expected to be produced in relativistic heavy ion collisions. Results are presented for CERN SPS NA49 $P b+P b$ experiment calculating hadron multiplicities and momentum spectra. The freeze-out properties of different hadrons are characterized by similar temperature, density and flow profiles in cylindrically symmetric geometry.
\end{abstract}

${ }^{a}$ The talk was presented by J. Zimányi at the Strangeness'96 Workshop, 15-17 May 1996, Budapest, Hungary. It will be published in the Proceedings of the Workshop, Heavy Ion Physics 4 (1996) 15.

\section{Introduction}

The evolution of hadronic fireball was successfully described by the hadrochemical model [1] in medium energy heavy ion collisions. This model is based on elementary processes in which hadron-hadron collisions lead to the production of other type of hadrons. The time-evolution of the hadronic system was described by a set of coupled differential equations in which the time-dependent gain and loss terms were determined by means of microscopical cross-sections for the elementary processes. The microscopical cross sections could be calculated in various approximations (e.g. considering free cross section, or medium effects or mean field, etc.), but the framework of the hadrochemistry calculation remains unaffected.

On the basis of the success of the hadrochemistry the quarkochemistry model [2] was developed to describe the evolution of quark matter produced at ultrarelativistic energies. Here the constituents of the quark-gluon plasma became the ingredients of the microscopical processes and the cross sections could be obtained in various ways (e.g. from perturbative QCD).

In the present paper we introduce a model in which the elementary processes contain the constituents of the semi-deconfined quark matter as incoming particles and the constituents of the hadron gas as outgoing particles. In this way the elementary processes of the phase transition from quark phase to hadron phase can be modeled. This model, which is essentially an extension of the ALCOR model [3] [4] may be called transchemistry, i.e. the chemical-like description of the phase transition. 
The basic assumption is the presence of the semi-deconfined quark matter. This means that gluons already disappeared from the system (they decayed or were absorbed), the main degrees of freedoms are quarks and antiquarks in the deconfined phase, they are dressed and ready for hadronization. One can use various approximations in the calculation of the cross sections for the elementary processes. Even more, one can include new terms into the set of the coupled differential equations to describe different collective properties of the system, e.g. temperature, flow or the strength of condensations, etc.

If we make a linearized approximation to the above mentioned set of differential equations - assuming short time durations only - then we arrive to the Algebraic Coalescence Rehadronization (ALCOR) model as presented in Ref. [3]. The ALCOR model is very successful to describe the hadron yields produced in different high energy heavy ion collisions 沺. Both transchemistry and ALCOR model yield particle numbers in the reaction volume $V$ which can be compared with the experimental results. However a more complete comparison can be developed if we know the full momentum distribution of the out-coming hadrons. Since $\sigma$, the production (hadronization) cross section has its maximum when the relative momentum of the participants is small (see later eq.(23), then we may assume that the spectrum of the produced hadrons can be characterized similarly as that of the quarks. Although a direct calculation of the momentum spectra of the out-coming hadrons is in progress, which is based on the microscopic coalescence applied in ALCOR [5], in the present paper we use the approximation mentioned before. Since the hadrons will suffer inelastic and elastic collisions during the time-evolution of the hadronic fireball, one can assume that all hadrons can be characterized with the same local temperature (or with the same temperature profile in case of an extended particle system). Because of the presence of a quark matter phase before hadronization one can assume that the collective flow profile will also be the same for the different hadron species. Furthermore, we will assume, that all hadrons can be characterized by the same density profile. The collective behaviour combined with the particle numbers obtained from transchemistry or ALCOR yield the necessary constrains to obtain the momentum spectra for all hadrons if we determined one of them (e.g. the spectrum of pions) from the experimental results.

In Section 2 the basic equations of transchemistry will be summarized and we show, how the former version of the ALCOR model can be derived from the equations of transchemistry. In Section 3 we display the ALCOR model, emphasizing its main properties. In Section 4 the hadronization rate will be introduced in the presence of flow which is a general result for particle systems characterized by a flow pattern. In Section 5 we display the particle numbers obtained from ALCOR for the $\mathrm{Pb}+\mathrm{Pb}$ collision at SPS energy. We also calculate the fully equilibrated hadron gas scenario and we discuss the main differences between these models. In Section 6 we generate momentum distributions for pion and proton spectra and compare with the experimental results in the $\mathrm{Pb}+\mathrm{Pb}$ collision. 


\section{Transchemistry and ALCOR}

In this section we will summarize the basic equations of transchemistry. For simplicity let us assume, that the net baryon charge of the system is zero and only $\pi$ mesons are produced. Thus the rate-equations for pion production from semideconfined quark matter are the following:

$$
\frac{d}{d t}\left[V(t) n_{\pi}(t)\right]=D^{(\pi)}\left\langle\sigma_{q \bar{q}}^{\pi} v\right\rangle_{(t)} \cdot n_{q}(t) \cdot n_{\bar{q}} V(t)-n_{\pi}(t) \cdot V(t) \cdot \Gamma_{\pi}
$$

The rate equations can be expressed in terms of particle numbers, too.

$$
\frac{d}{d t} N_{\pi}(t)=\frac{D^{(\pi)} \cdot\left\langle\sigma_{q \bar{q}}^{\pi} v\right\rangle_{(t)}}{V(t)} \cdot N_{q}(t) \cdot N_{\bar{q}}(t)-N_{\pi}(t) \cdot \Gamma_{\pi},
$$

One obtains similar equations for the quark and antiquark numbers:

$$
\begin{aligned}
\frac{d}{d t} N_{q}(t) & =-\frac{D^{(\pi)} \cdot\left\langle\sigma_{q \bar{q}}^{\pi} v\right\rangle_{(t)}}{V(t)} \cdot N_{q}(t) \cdot N_{\bar{q}}(t)+N_{\pi}(t) \cdot \Gamma_{\pi} \\
\frac{d}{d t} N_{\bar{q}}(t) & =-\frac{D^{(\pi)} \cdot\left\langle\sigma_{q \bar{q}}^{\pi} v\right\rangle_{(t)}}{V(t)} \cdot N_{q}(t) \cdot N_{\bar{q}}(t)+N_{\pi}(t) \cdot \Gamma_{\pi}
\end{aligned}
$$

Here $n_{i}(t)$ denotes the density of particle $i, V(t)$ is the reaction volume within which constant density was assumed and $N_{i}(t)$ is the number of particle $i$. The $\Gamma_{\pi}$ denotes the loss term for pions related to their 'decay' (or re-entering the semideconfined quark matter). The $\left\langle\sigma_{q \bar{q}}^{\pi} v\right\rangle_{(t)}$ denotes the momentum averaged elementary hadronization rate for one hadronic degree of freedom and $D^{(\pi)}$ is the spin degeneracy factor for the final hadron. The temperature, density and flow profile for the quark distributions inside the volume $V$ can be chosen in various ways. We will assume that they are identical with the hadronic ones obtained from the experiments. The hadronization rate can be calculated in many microscopic ways assuming different models: quark coalescence [3], Nambu-Jona-Lasinio model [6], or other effective quark models, e.g. [7].

From eq.(2)-(3)-(4) one obtains that $N_{\pi}+N_{q}=$ const. and $N_{\pi}+N_{\bar{q}}=$ const., which is the number conservation for the hadronization process $q+\bar{q} \Longleftrightarrow \pi$. If the microscopical hadronization mechanism is different, e.g. $q+\bar{q} \Longleftrightarrow \pi+\pi$, then the above equations will contain different coefficients. In its recent form transchemistry equations conserve not only baryon numbers, but the numbers of the constituent quarks and antiquarks.

In general case the particle number production for meson $M$ can be described as follows:

$$
\frac{d}{d t} N_{M}\left(q_{i}, \bar{q}_{j} ; t\right)=\frac{D^{(M)}\left\langle\sigma_{q_{i} \bar{q}_{j}}^{M} v\right\rangle_{(t)}}{V(t)} N_{q_{i}}(t) N_{\bar{q}_{j}}(t)-\sum N_{M}\left(q_{i}, \bar{q}_{j} ; t\right) \Gamma_{M}
$$


In the loss term the $\sum$ means all of the possible decay channels. We plan to apply the transchemistry for fast hadronization processes, in which case the deconfinement channels have minor role. Thus we will neglect the decay channels in the followings.

If the hadronization starts at time $t_{0}$ and ends at time $t_{0}+\tau$, then one can obtain the produced hadron numbers from the following integral:

$$
N_{M}\left(q_{i}, \bar{q}_{j} ; t_{0}+\tau\right)=\int_{t_{0}}^{t_{0}+\tau} \frac{D^{(M)}\left\langle\sigma_{q_{i} \bar{q}_{j}}^{M} v\right\rangle_{(t)}}{V(t)} \cdot N_{q_{i}}(t) \cdot N_{\bar{q}_{j}}(t) d t
$$

In transchemistry various hadrons can be formed from the deconfined quark matter. Meson formation is relatively simple, because one step is enough to confine a quark-antiquark pair into meson-like hadron. Baryon (and antibaryon) production might happen in three-body interactions or sequentially via two-body interactions. In the latter case diquarks (anti-diquarks) will be produced and the timeevolution of their density should be followed also. The microscopical description of baryon production is not a well-developed field, so we use the simplest two-step processes to form baryons. In this case we need to follow the production of diquarks and anti-diquarks also, as they are carrying explicitly certain degrees of freedom in transchemistry.

In case of fast hadronization (e.g. an overcooled quark matter is converted into hadrons [8]) the volume $V$ and the averaged rate $\langle\sigma v\rangle$ will not change too quickly, thus one can apply the theorem of mean value of the integral calculus. For one integral this transcription is exact. The first term in eq. (6) - which contains the rate and the volume - remains approximately unchanged, but the time-dependent function of quark numbers will be substituted by their mean value at time $t_{i}^{*}\left(t_{0}<t_{i}^{*}<t_{0}+\tau\right.$, where $\left.\tau<<t_{0}\right)$ :

$$
N_{M}\left(q_{i}, \bar{q}_{j} ; t_{0}+\tau\right)=\frac{D^{(M)}\left\langle\sigma_{q_{i} \bar{q}_{j}}^{M} v\right\rangle \cdot \tau}{V} \cdot N_{q_{i}}\left(t_{i}^{*}\right) \cdot N_{\bar{q}_{j}}\left(t_{j}^{*}\right)
$$

One can approximate the particle numbers at $t^{*}$ by means of the initial particle numbers: $N_{q_{i}}\left(t_{i}^{*}\right) \approx b_{q_{i}} N_{q_{i}}\left(t_{0}\right)$ and $N_{\bar{q}_{j}}\left(t_{j}^{*}\right) \approx b_{\bar{q}_{j}} N_{\bar{q}_{j}}\left(t_{0}\right)$. If the parameters $b_{q_{i}}$ and $b_{\bar{q}_{j}}$ were time-independent then the above approximation can be useful to simplify the time-dependent transchemistry equations:

$$
N_{M}\left(q_{i}, \bar{q}_{j} ; t_{0}+\tau\right)=\frac{D^{(M)}\left\langle\sigma_{q_{i} \bar{q}_{j}}^{M} v\right\rangle \cdot \tau}{V} \cdot b_{q_{i}} N_{q_{i}}\left(t_{0}\right) \cdot b_{\bar{q}_{j}} N_{\bar{q}_{j}}\left(t_{0}\right)
$$

The complete set of time dependent differential equations of the type of eq. (5) preserve all particle numbers which should be preserved - as we mentioned earlier. However, after the above linear approximation the conserved particle numbers will not be preserved automatically. Therefore we have to impose the conservation law of particle numbers (namely quark and antiquark numbers) while keeping the essence of the above set of equations. In this way one arrives to the basic equations of the phenomenological ALCOR model, as a linearized, averaged special case of the transchemistry. 


\section{The ALCOR model}

The starting point of the ALCOR model [3] [1] is a semi-deconfined state in which only those quarks and antiquarks are present in local thermal equilibrium which form the final hadrons. We also assume that only quarks and antiquarks are present, so their number already accounts for those gluons which were fragmented earlier. This assumption is easily acceptable for the heavy quarks for which any meeting with their antiparticle is improbable due to their small specific density. In the case of light constituent quarks (counted after gluon fragmentation) the approximate entropy conservation during the rehadronization process leads to the conservation of their numbers. This picture was first formulated in the framework of the algebraic recombination model [9]. In this model the number of a given type of hadron produced is proportional to the product of the numbers of their constituting quarks. This picture was developed further by introducing gluon fragmentation [10]. We shall denote the number of produced $u, d, s$ quark pairs - just before the hadronization - by $N_{u, p a i r}, N_{d, p a i r}$ and $N_{s, p a i r}$, respectively. The strangeness production factor, $g_{S}$ is defined as $g_{S}=N_{s, \text { pair }} /\left(N_{u, \text { pair }}+N_{d, \text { pair }}\right)$.

The mesonic coalescence factors of the ALCOR model can be introduced by means of eq. (8), namely

$$
C_{M}\left(q_{i}, \bar{q}_{j}\right)=\frac{\left\langle\sigma_{q_{i} \bar{q}_{j}}^{M} v\right\rangle \cdot \tau}{V}
$$

Thus the production of meson $M$ can be described as follows:

$$
N_{M}\left(q_{i}, \bar{q}_{j}\right)=D^{(M)} C_{M}\left(q_{i}, \bar{q}_{j}\right) \cdot b_{q_{i}} N_{q_{i}} \cdot b_{\bar{q}_{j}} N_{\bar{q}_{j}}
$$

The baryonic (antibaryonic) coalescence factors can be defined and calculated as a simple sequential process, when baryons containing three constituent quarks will be produced from diquarks and quarks. The coalescence factors are the following:

$$
\begin{aligned}
C_{B}(a, b, c)= & g_{B} \frac{1}{3}\left\{C_{M}(a, b) C_{M}([a+b], c)+\right. \\
& \left.+C_{M}(a, c) C_{M}([a+c], b)+C_{M}(b, c) C_{M}([b+c], a)\right\} .
\end{aligned}
$$

Here indices $a, b, c$ means the $q_{i}$ quarks for baryon production or $\bar{q}_{i}$ antiquarks for antibaryon production. The factor $g_{B}$ is the baryon suppression factor. Its physical meaning can be connected to the sequential creation of the baryons. We assume that the production of a baryon from three quarks or an antibaryon from three antiquarks is a two-step process leading through an intermediate diquark formation in the corresponding color triplet or anti-triplet state which forms the final baryon later on together with a third quark or antiquark. These diquarks must, however, be very unstable, short-lived clusters, so they may decay before forming the baryon. We take into account this mechanism of baryon production by introducing the phenomenological baryon suppression parameter, $g_{B}$. Mathematically one can introduce the factor $g_{B}$ similarly as the parameter $b_{i}$, namely approximating 
the time-dependent integrals for baryon production with the substitution of mean integral values. However, $g_{B}$ seems to be a well-behaving physical parameter, so we will determine it from the experimental results, similarly to $g_{S}$. We will assume that $g_{B}$ is flavour blind.

Now one can determine the numbers of the baryons and antibaryons:

$$
\begin{aligned}
& N_{B}\left(q_{i}, q_{j}, q_{k}\right)=D^{(B)} C_{B}\left(q_{i}, q_{j}, q_{k}\right) \cdot b_{q_{i}} N_{q_{i}} \cdot b_{q_{j}} N_{q_{j}} \cdot b_{q_{k}} N_{q_{k}}, \\
& N_{\bar{B}}\left(\bar{q}_{i}, \bar{q}_{j}, \bar{q}_{k}\right)=D^{(\bar{B})} C_{\bar{B}}\left(\bar{q}_{i}, \bar{q}_{j}, \bar{q}_{k}\right) \cdot b_{\bar{q}_{i}} N_{\bar{q}_{i}} \cdot b_{\bar{q}_{j}} N_{\bar{q}_{j}} \cdot b_{\bar{q}_{k}} N_{\bar{q}_{k}} .
\end{aligned}
$$

In the above equations the spin degeneracies are also included, $D^{(H)}=2 S_{H}+1$. Here we shall consider the simplest hadron multiplets conserving isospin symmetry. These are the spin 0 and spin 1 meson octets together with the $\sigma$ and $\omega$ isospin singlet meson states. Furthermore the spin $1 / 2$ octet and spin $3 / 2$ decouplet baryons and anti-baryons are also included.

Since we have introduced the numbers of different mesons, baryons and antibaryons produced from the quark matter, one has to put up the final set of equations, which will ensure the conservation of the numbers of different flavours (for each quark and each antiquark flavor):

$$
\begin{aligned}
& N_{q_{i}}=\sum_{H} \sum_{j=1}^{N_{f}} D^{(M)} C_{M}\left(q_{i}, \bar{q}_{j}\right) \cdot b_{q_{i}} N_{q_{i}} \cdot b_{\bar{q}_{j}} N_{\bar{q}_{j}} \\
& +\sum_{H} \sum_{j=1}^{N_{f}} \sum_{k=j}^{N_{f}}\left(1+\delta_{i, j}+\delta_{i, k}\right) D^{(B)} C_{B}\left(q_{i}, q_{j}, q_{k}\right) \cdot b_{q_{i}} N_{q_{i}} \cdot b_{q_{j}} N_{q_{j}} \cdot b_{q_{k}} N_{q_{k}} \\
& N_{\bar{q}_{i}}=\sum_{H} \sum_{j=1}^{N_{f}} D^{(M)} C_{M}\left(\bar{q}_{i}, q_{j}\right) \cdot b_{\bar{q}_{i}} N_{\bar{q}_{i}} \cdot b_{q_{j}} N_{q_{j}} \\
& +\sum_{H} \sum_{j=1}^{N_{f}} \sum_{k=j}^{N_{f}}\left(1+\delta_{i, j}+\delta_{i, k}\right) D^{(\bar{B})} C_{\bar{B}}\left(\bar{q}_{i}, \bar{q}_{j}, \bar{q}_{k}\right) \cdot b_{\bar{q}_{i}} N_{\bar{q}_{i}} \cdot b_{\bar{q}_{j}} N_{\bar{q}_{j}} \cdot b_{\bar{q}_{k}} N_{\bar{q}_{k}} \cdot(1)
\end{aligned}
$$

This set of equations determines uniquely the normalization factors, $b_{i}$. The number of independent equations in eqs.(14), (15) is equal to the number of independent $b_{q_{i}}$ and $b_{\bar{q}_{i}}$ factors. Note, that these $2 N_{f}$ quantities are not adjustable parameters of the model, but they are determined by the flavour conservation.

Furthermore, introducing the factors $b_{q_{i}}$ and $b_{\bar{q}_{i}}$ we have constructed a scale invariant description for the particle production. Namely, the physical quantities can be transformed in the following way:

$$
\begin{aligned}
C_{M}\left(\bar{q}_{i}, q_{j}\right) & \longrightarrow \lambda_{i} \bar{\lambda}_{j} C_{M}\left(\bar{q}_{i}, q_{j}\right), \\
C_{B}\left(q_{i}, q_{j}, q_{k}\right) & \longrightarrow \lambda_{i} \lambda_{j} \lambda_{k} C_{B}\left(q_{i}, q_{j}, q_{k}\right), \\
C_{\bar{B}}\left(\bar{q}_{i}, \bar{q}_{j}, \bar{q}_{k}\right) & \longrightarrow \bar{\lambda}_{i} \bar{\lambda}_{j} \bar{\lambda}_{k} C_{\bar{B}}\left(\bar{q}_{i}, \bar{q}_{j}, \bar{q}_{k}\right), \\
b_{q_{i}} & \longrightarrow b_{q_{i}} / \lambda_{i}, \\
b_{\bar{q}_{i}} & \longrightarrow b_{\bar{q}_{i}} / \bar{\lambda}_{i}
\end{aligned}
$$


Substituting the results of this transformation into eqs. (10), (12), (13), one can see that the numbers of produced hadrons, $N_{M}\left(q_{i}, \bar{q}_{j}\right), N_{B}\left(q_{i}, q_{j}, q_{k}\right)$ and $N_{\bar{B}}\left(\bar{q}_{i}, \bar{q}_{j}, \bar{q}_{k}\right)$ remain unchanged. Thus even if we miss a constant factor in the microscopical hadronization coefficients, then one can obtain the particle numbers correctly.

\section{Hadronization Rate in the Presence of Flow}

In the presence of a flow we consider the relativistic Jüttner distribution

$$
f(x, p)=e^{-\beta p \cdot u(x)}
$$

with $u_{\mu}(x)$ being the local four-velocity of the flow. Let us restrict ourselves in the following to a scaling longitudinal flow 11. In this case the thermal averaging of the rate can be interpreted only locally: the earlier rate equations has to be reinterpreted in terms of reacting components from different coordinate rapidity ranges $\eta_{a}$ and $\eta_{b}$ respectively

$$
\frac{1}{V} \frac{d}{d \tau}\left(V n_{h}\right)=\int d \eta_{a} d \eta_{b}\langle\sigma v\rangle_{a b} n_{a} n_{b} .
$$

Clearly the total volume of the fireball increases due to a longitudinal scaling expansion like $V=\tau \pi R^{2} \Delta \eta$ with total coordinate rapidity extension $\Delta \eta$ and transverse radius $R$. Assuming a space-time rapidity plateau for a finite interval in the number distribution of the reacting and produced components we arrive at a modified rate equation

$$
\tau \frac{d}{d \tau} N_{h}=\frac{\lambda^{*}}{\pi R^{2}} N_{a} N_{b}
$$

Here

$$
\lambda^{*}=\int d\left(\eta_{a}-\eta_{b}\right)\langle\sigma v\rangle \Theta\left(\left(p_{a}-p_{b}\right) \cdot\left(x_{a}-x_{b}\right)\right)
$$

is the total rate of reactions between all possible coordinate rapidity cell pairs. The constraint $\left(p_{a}-p_{b}\right) \cdot\left(x_{a}-x_{b}\right) \geq 0$ reduces to the requirement that the relative velocity vector is oriented opposite to the relative position vector in the center of mass system of the colliding components 'a' and 'b'. This constraint is necessary because only those particles collide which have a relative velocity pointing towards each other.

The inclusion of this constraint after some algebraic manipulation leads to the general expression of the averaged rate

$$
\lambda^{*}=\frac{\beta \int d \sqrt{s} \sigma(s) \lambda_{a b}^{*}(s) G(\beta \sqrt{s})}{8 m_{a}^{2} m_{b}^{2} K_{2}\left(\beta m_{a}\right) K_{2}\left(\beta m_{b}\right)}
$$


where $\lambda_{a b}^{*}(s)=\left[s-\left(m_{a}+m_{b}\right)^{2}\right]\left[s-\left(m_{a}-m_{b}\right)^{2}\right]$ and $G(\beta \sqrt{s})$ is the thermal weight factor. In the presence of longitudinal scaling flow the latter can be written as an integral over relative coordinate rapidity $\eta=\left(\eta_{a}-\eta_{b}\right)$

$$
G(\beta \sqrt{s})=\int_{-\infty}^{\infty} d \eta \sqrt{s} \frac{K_{0}(\beta \sqrt{s} \operatorname{ch}|\eta|)-K_{0}\left(\beta \sqrt{s} \operatorname{ch}|\eta|+\beta \frac{\sqrt{\lambda_{a b}^{*}(s)}}{2 \sqrt{s}} \operatorname{sh}|\eta|\right)}{\beta \sqrt{\lambda_{a b}^{*}(s)} \operatorname{ch}|\eta| \operatorname{sh}|\eta|} .
$$

Investigating the integrand of eq. 22 one observes a finite width, $\delta \eta$, in the relative coordinate rapidity $\eta$. (This quantity, $\delta \eta$, is equal to the relative flow rapidity because of the Bjorken scaling assumption.) The finite width depends on temperature $T$, on the particle rest masses $m_{a}, m_{b}$, and on the considered energy scale $\sqrt{s}$ in a complicated manner.

The most uncertain ingredient of the ALCOR hadronization model is the hadronization cross section in eq. (21). We will consider an analogy with the $p+A \rightarrow$ $d+(A-1)$ nuclear rearrangement (pick-up) reaction leading to deuteron formation [12. Assuming a Coulomb-like potential in the final $(q \bar{q})$ state a fusing cross section can be derived from this analogy:

$$
\sigma=16 m_{h}^{2} \sqrt{\pi} \rho^{3} \frac{\alpha^{2} a}{\left(1+(k a)^{2}\right)^{2}}
$$

Here $m_{h}$ is the rest mass of the meson, while $a=1 /\left(m_{a b} \alpha\right)$ is the Bohr radius of the bound $q \bar{q}$ state in a $V(r)=-\alpha / r$ Coulomb potential with $m_{a b}$ being the reduced mass of particles 'a' and 'b'. In our calculation we took $\alpha=0.46$ and $T=200$ $\mathrm{MeV}$ temperature in estimating hadronization cross sections at CERN SPS energy. The factor $\rho=0.3 \mathrm{fm}$ occurring in eq. 23) accounts for the medium influencing the hadron formation and was taken to be equal to the Debye screening length in quark-gluon plasma at the above temperature. Finally $k$ occurring in eq.(23) is the magnitude of the relative momentum vector of particles 'a' and 'b' measured in their center of mass system $k=\sqrt{\lambda_{a b}^{*}(s)} / 2 \sqrt{s}$.

\section{$5 \quad$ Results for $\mathrm{Pb}+\mathrm{Pb}$ collision from $\mathrm{ALCOR}$}

Well armed with the above theoretical considerations the redistribution of different flavor quarks and antiquarks into all possible hadrons can be calculated in the ALCOR model using practically only three parameters: i) the total number of quarkantiquark pairs, $N_{\text {tot,pair }}=N_{u, \text { pair }}+N_{\text {d,pair }}+N_{s, \text { pair }}$, which can be determined from the measured total charged multiplicity, ii) the parameter $g_{B}$ controlling the baryon formation and iii) the strangeness production factor $g_{S}$. The dependence of the final results on the other parameters (e.g. $T, \alpha)$ within their physically acceptable interval is small. All further results, such as the number of hyperons, kaons, etc. are predictions of the ALCOR model. 
In Ref. [4] we presented our results for the $\mathrm{S}+\mathrm{S}$ collisions at $200 \mathrm{GeV} /$ nucleon bombarding energy. We took into account that the number of participant nucleons was measured to be $N_{\text {partic }}^{S S}=51$ [13]. We used the parameters $N_{\text {tot,pair }}^{S S}=158.1$ with $g_{S}=0.255$ for the strangeness production, and $g_{B}=0.04$ for the baryon formation. The obtained particle numbers fitted the experimental results excellently.

From this experience we conjectured that in $\mathrm{S}+\mathrm{S}$ collision the rehadronization process is locally quick: the quarks and antiquarks (including the fragmented gluons) appear in hadrons according to simple kinematic rules and production branching ratios. This finding, however, does not settle the question whether quark matter with any degree of collectivity has been formed or other hadronization processes including strings or color ropes [14] are the source of this amount of quarks. Local thermal distribution of the different quark flavors and the presence of a longitudinal flow cannot, on the other hand, be excluded on the basis of this experimental data.

To obtain results for $\mathrm{A}+\mathrm{A}$ collisions we assume a scaling connected to the participant nucleon number for the produced quark-antiquark pairs:

$$
N_{\text {tot }, \text { pair }}^{A A}(\sqrt{s})=\left(\frac{N_{\text {partic }}^{A A}}{N_{\text {partic }}^{S S}}\right)^{\alpha} N_{\text {tot,pair }}^{S S}(\sqrt{s}),
$$

where $N_{\text {partic }}^{A A}$ are the number of participant nucleons in the $\mathrm{A}+\mathrm{A}$ collision. The scaling exponent $\alpha$ may have the value $\alpha=1$, or, for more collective production processes one expects $\alpha>1$. Furthermore we shall use the values for $g_{B}$ and $g_{S}$ obtained above.

At the extrapolation of the total number of produced quark-antiquark pairs from the $\mathrm{S}+\mathrm{S}$ collision one has to consider an energy scaling. We will assume a logarithmic one:

$$
\frac{N_{\text {tot,pair }}^{A A}\left(\sqrt{s_{1}}\right)}{N_{\text {tot }, \text { pair }}^{A A}\left(\sqrt{s_{2}}\right)}=\frac{\ln \sqrt{s_{1}}}{\ln \sqrt{s_{2}}}
$$

In our case, considering $160 \mathrm{GeV} /$ nucleon collision energy for the $\mathrm{Pb}+\mathrm{Pb}$ collision, this rescaling yields an $\approx 4 \%$ correction for the $N_{t o t, p a i r}^{P b P b}$.

Now we can make prediction for the total particle production in the $\mathrm{Pb}+\mathrm{Pb}$ collision. The number of participant nucleons is $N_{\text {part }}^{P b P b}=390 \pm 10$ obtained from Monte-Carlo simulations. Using the mean value and a linear scaling $(\alpha=1)$ one obtains $N_{\text {tot,pair }}^{P b P b}=1164$. We keep the strangeness production and baryon formation factors fitted for $\mathrm{S}+\mathrm{S}$ collision, $g_{S}=0.255, g_{B}=0.04$. Table 1 shows our prediction for the particle numbers together with the results of two thermal equilibrium models, Thermal I and Thermal II. In Table 2 some characteristic particle ratios are displayed for the three models. 
Table 1. Particle numbers: prediction of ALCOR model for the $\mathrm{Pb}+\mathrm{Pb}$ collision and the results of full equilibrium thermal models, 'Thermal I-II'.

\begin{tabular}{||c||c|c|c||}
\hline \hline Pb+Pb & ALCOR & Thermal I. & Thermal II. \\
\hline \hline$h^{-}$ & 730.41 & 798.72 & 839.44 \\
\hline \hline$\pi^{+}$ & 603.87 & 603.97 & 603.54 \\
\hline$\pi^{0}$ & 618.95 & 620.70 & 619.16 \\
\hline$\pi^{-}$ & 634.68 & 637.91 & 635.31 \\
\hline$K^{+}$ & 84.15 & 174.57 & 205.68 \\
\hline$K^{0}$ & 84.15 & 177.74 & 211.29 \\
\hline $\bar{K}^{0}$ & 41.65 & 103.10 & 119.46 \\
\hline$K^{-}$ & 41.65 & 104.86 & 122.69 \\
\hline$K_{S}^{0}$ & 62.90 & 140.42 & 165.38 \\
\hline \hline$p^{+}$ & 170.90 & 141.58 & 129.97 \\
\hline$n^{0}$ & 188.57 & 148.20 & 133.44 \\
\hline$\Sigma^{+}$ & 12.86 & 24.21 & 41.27 \\
\hline$\Sigma^{0}$ & 13.63 & 25.04 & 42.37 \\
\hline$\Sigma^{-}$ & 14.43 & 25.89 & 43.51 \\
\hline$\Lambda^{0}$ & 68.20 & 55.87 & 42.37 \\
\hline$\Xi^{0}$ & 8.82 & 10.92 & 11.99 \\
\hline$\Xi^{-}$ & 8.89 & 11.09 & 12.31 \\
\hline$\Omega^{-}$ & 1.48 & 1.56 & 2.92 \\
\hline \hline $\bar{p}^{-}$ & 25.07 & 13.28 & 14.57 \\
\hline $\bar{n}^{0}$ & 25.07 & 12.68 & 14.19 \\
\hline $\bar{\Sigma}^{-}$ & 4.18 & 4.10 & 8.10 \\
\hline $\bar{\Sigma}^{0}$ & 4.18 & 3.96 & 7.89 \\
\hline $\bar{\Sigma}^{+}$ & 4.18 & 3.83 & 7.68 \\
\hline $\bar{\Lambda}^{0}$ & 20.93 & 8.84 & 7.89 \\
\hline $\bar{\Xi}^{0}$ & 5.98 & 3.10 & 3.91 \\
\hline $\bar{\Xi}^{+}$ & 5.98 & 3.05 & 3.81 \\
\hline $\bar{\Omega}^{+}$ & 2.20 & 0.77 & 1.59 \\
\hline \hline & & & \\
\hline
\end{tabular}


Table 2. Particle ratios: prediction of ALCOR model for the $\mathrm{Pb}+\mathrm{Pb}$ collision and the results of full equilibrium thermal models, 'Thermal I-II'.

\begin{tabular}{||c||c|c|c||}
\hline \hline $\mathbf{P b}+\mathbf{P b}$ & ALCOR & Thermal I. & Thermal II. \\
\hline \hline$p^{+} / \pi^{+}$ & 0.28 & 0.23 & 0.21 \\
\hline $\bar{p}^{-} / p^{+}$ & 0.14 & 0.09 & 0.11 \\
\hline $\bar{p}^{-} / \pi^{-}$ & 0.04 & 0.02 & 0.02 \\
\hline$K^{+} / \pi^{+}$ & 0.14 & 0.29 & 0.34 \\
\hline$K^{+} / K-$ & 2.02 & 1.66 & 1.67 \\
\hline$K_{S}^{0} / \Lambda^{0}$ & 0.92 & 2.51 & 3.90 \\
\hline $\bar{\Lambda}^{0} / \bar{p}^{-}$ & 0.83 & 0.66 & 0.54 \\
\hline $\bar{\Lambda}^{0} / \Lambda^{0}$ & 0.30 & 0.16 & 0.18 \\
\hline$\Xi^{-} / \Lambda^{0}$ & 0.13 & 0.19 & 0.29 \\
\hline $\bar{\Xi}^{+} / \bar{\Lambda}^{0}$ & 0.28 & 0.34 & 0.48 \\
\hline$\Xi^{-} / \bar{\Xi}^{+}$ & 1.48 & 3.62 & 3.22 \\
\hline $\bar{p}^{-} / K^{-}$ & 0.60 & 0.13 & 0.12 \\
\hline$\Lambda^{0} / K^{-}$ & 1.64 & 0.53 & 0.35 \\
\hline \hline
\end{tabular}

In the thermal models we assume a homogeneous system in full chemical equilibrium at different level. In model 'Thermal-I' the same hadron multiplets are assumed to be in equilibrium, which are populated in the ALCOR model, and then the short lived resonances are allowed to decay into the stable particles shown in the Tables. On the other hand, for the model 'Thermal-II' we assumed, that the final (stable) particles are in chemical equilibrium. In both cases the values were determined to get approximately the same $\pi^{+}$number as in ALCOR at a given temperature, $T=165 \mathrm{MeV}$. The chemical potential for strange quarks were determined to reproduce strangeness neutrality. The light quark chemical potential was obtained from the total baryon number of the system.

Comparing the results from ALCOR with the results from the thermal model, we find that, surprisingly enough, they are not far from each other. The greatest deviation is about a factor of five in some of the particle ratios (see e.g. $\bar{p}^{-} / K^{-}$).

This similarity is due, probably, to the fact that, i) we have a large number of resonances; ii) the spin degeneracy factor appears in both models; iii) the conservation rules lead to strong restrictions.

Besides the similarities there are systematic differences between the two models. The strange baryon to strange meson ratio in the thermal model is systematically smaller than in the ALCOR results. This can be interpreted as the effect of mass differences, which leads to an $\exp (-\mathrm{m} / \mathrm{T})$ suppression in the thermal case, while it is not present in ALCOR. Thus this ratio may indicate that how strong was the hadrochemical evolution in the final hadronic phase. 


\section{Momentum distribution}

In the ALCOR model we have a microscopical dynamical prescription for the process of quark coalescence into hadrons. Furthermore we assumed a given momentum distribution for the constituent quarks. Thus the momentum distribution of the produced hadrons can be calculated. However, this is a somewhat tedious procedure. On the other hand we observe, that the capture cross section ( eq.(23) ) has its maximum at zero relative quark momentum. Therefore one may assume, that the hadrons, just after their production will have a momentum distribution similar to that of the quarks ( eq.(17) ). In this chapter, however, for the hadrons we shall use Fermi and Bose distributions, and a transverse flow is added, too (see eq.(28) below). We assume, that, due to the elastic collisions, all the hadrons will have the same density, flow and temperature profiles. However, the particle composition will remain the same as it was at the hadronization. Thus we have chemically not equilibrated system, where all hadrons will have its own chemical potential, corresponding to the partial density of this species. The value of the temperature, $T$, the transverse radius, $R$, the radial flow velocity at the surface and the proper time, $\tau$, are determined by fitting the measured rapidity and transverse mass distribution. However, due to our assumptions, the same set of parameters must be used for all type of particles. Thus the existence of such a self-consistent fit is not trivial! From the momentum distributions and the volume we can calculate the entropy of the system, too.

In general the invariant momentum distribution will be the following:

$$
E \frac{d^{3} N}{d^{3} p}=\frac{1}{(2 \pi \hbar c)^{3}} \int \frac{g_{d e g}}{e^{\left(p^{\nu} u_{\nu}-\mu\right) / T}+K} p^{\nu} d \sigma_{\nu}
$$

We will keep the quantum statistics, so for mesons we have $K=-1$, for baryons and antibaryons $K=1$. Later we will see the importance of quantum statistics for pions. For correct freeze-out calculation we need to introduce the Cooper Frye pre-factor $p^{\nu} d \sigma_{\nu}$ [15]. Let us consider a cylindrically symmetric geometry and assume freeze-out at constant proper time $\tau$. For such a freeze-out hypersurface one obtains

$$
p^{\nu} d \sigma_{\nu}=m_{t} r d r \tau \cosh (y-\eta) d \eta d \phi_{r} .
$$

Furthermore, let us assume a longitudinal Bjorken flow and a linear self-similar transverse flow,

$$
u_{\nu}=\left[\cosh (\eta) \cosh (\Theta), \sinh (\eta) \cosh (\Theta), \sinh (\Theta) \cos \left(\phi_{r}\right), \sinh (\Theta) \sin \left(\phi_{r}\right)\right]
$$

where the transverse flow is defined as $v_{t}=\tanh (\Theta)$. The transverse radius depends on the proper time during expansion, namely it is $R(\tau)$. 
After trivial calculation one obtains the invariant momentum spectra:

$$
\frac{d N}{m_{t} d m_{t} d y}=\frac{\tau g_{d e g}}{2 \pi(\hbar c)^{3}} \int_{0}^{R(\tau)} r d r \int_{\eta_{\min }}^{\eta_{\max }} d \eta \int_{0}^{\pi} d \phi \frac{m_{t} \cosh (y-\eta)}{e^{\left(p^{\nu} u_{\nu}-\mu\right) / T}+K}
$$

where $\phi=\phi_{r}-\phi_{p}$ and $p^{\nu} u_{\nu}=m_{t} \cosh (\Theta) \cosh (y-\eta)-p_{t} \sinh (\Theta) \cos \phi$.

The calculation of particle spectra is based on the eq. (29), where the thermodynamical parameters depend on the radius, $T(r)$ and $\left.\mu_{(} r\right)$. Considering Bjorken scaling, we neglect here longitudinal dependence, only the finite longitudinal size of the fireball is included, $\left[z_{\min }, z_{\max }\right]=\left[\tau \cdot \sinh \eta_{\min }, \tau \cdot \cosh \eta_{\min }\right]$.

If we investigate the radial dependence of the particle numbers, we need to introduce the following distribution function.

$$
\frac{d N}{r d r}=\frac{\tau g_{d e g}}{2 \pi(\hbar c)^{3}} \int_{\eta_{\min }}^{\eta_{\max }} d \eta \int_{-\infty}^{+\infty} d y \int_{m}^{+\infty} m_{t} d m_{t} \int_{0}^{\pi} d \phi \frac{m_{t} \cosh (y-\eta)}{e^{\left(p^{\nu} u_{\nu}-\mu\right) / T}+K}
$$

We assume that these radial distributions are similar for different particle species at freeze-out time $\tau_{f}$.

The total energy of the system can be obtained from eq.(30) by multiplying the integral with $E=m_{t} \cosh y$ and integrating over $r$.

Before comparing the experimental results and the calculated particle spectra, let us summarize shortly the possible values of different parameters. For the temperature profile no longitudinal dependence was considered, the radial dependence was chosen to be quadratic or higher, $T(\eta, r, \tau)=T_{0}(\tau) \cdot\left[1-(r / R(\tau))^{\gamma}\right]$, $T_{0}\left(\tau_{f}\right)=150-180 \mathrm{MeV}, \gamma \geq 2$. There is a longitudinal Bjorken scaling and for the transverse flow we will use the parameterization $v_{T}(r, \tau)=\tanh (\Theta(r, \tau))$, where $\sinh (\Theta(r, \tau))=w_{T}(\tau) \cdot r / R(\tau)$ and on the radial surface at freeze out time it is $v_{T}\left(R\left(\tau_{f}\right), \tau_{f}\right)=w_{T}\left(\tau_{f}\right) / \sqrt{w_{T}^{2}\left(\tau_{f}\right)+1}<1$. The transverse radius, $R\left(\tau_{f}\right)$, the minimal and maximal value of the space-time rapidity, $\left(\eta_{\min }, \eta_{\max }\right)$ and the proper time of the freeze-out $\tau_{f}$ are free parameters $\left(z_{\min }=\tau \sinh \eta_{\min }\right.$, $\left.z_{\max }=\tau \sinh \eta_{\max }\right)$. Chemical potentials, $\mu(r)$ will be calculated from $\mathrm{dN} / \mathrm{rdr}$.

Fig. 1 and 2 display the calculated $\pi^{-}$and proton spectra at $\tau_{f}=6 \mathrm{fm}$, $T_{0}\left(\tau_{f}\right)=165 \mathrm{MeV}, \gamma=2, \eta_{\min , \max }= \pm 1.75$ (which means longitudinal size $|z|= \pm 16.75 \mathrm{fm}), R_{T}\left(\tau_{f}\right)=14 \mathrm{fm}, v_{T}\left(R\left(\tau_{f}\right)\right)=0.77$. In Fig. 2 the full lines indicate Bose and Fermi distributions, the dotted line indicates results for pion with Boltzmann distribution. The transverse momentum distribution of pions shown in Fig. 2. suggests that the Bose statistics plays a role in the enhancement of the low $m_{t}$ part of the pion spectra. Such an effect was suggested earlier for the heavy ion collisions at Bevatron [16]. If we increase the freeze out volume by a factor of two, the pion density decreases, and the low $m_{t}$ enhancement disappears.

The total entropy and energy of the hadron system were discussed in Ref. [17], where it was obtained $S / N_{B}=30$ for the specific entropy in the above parametrized hadron gas. 

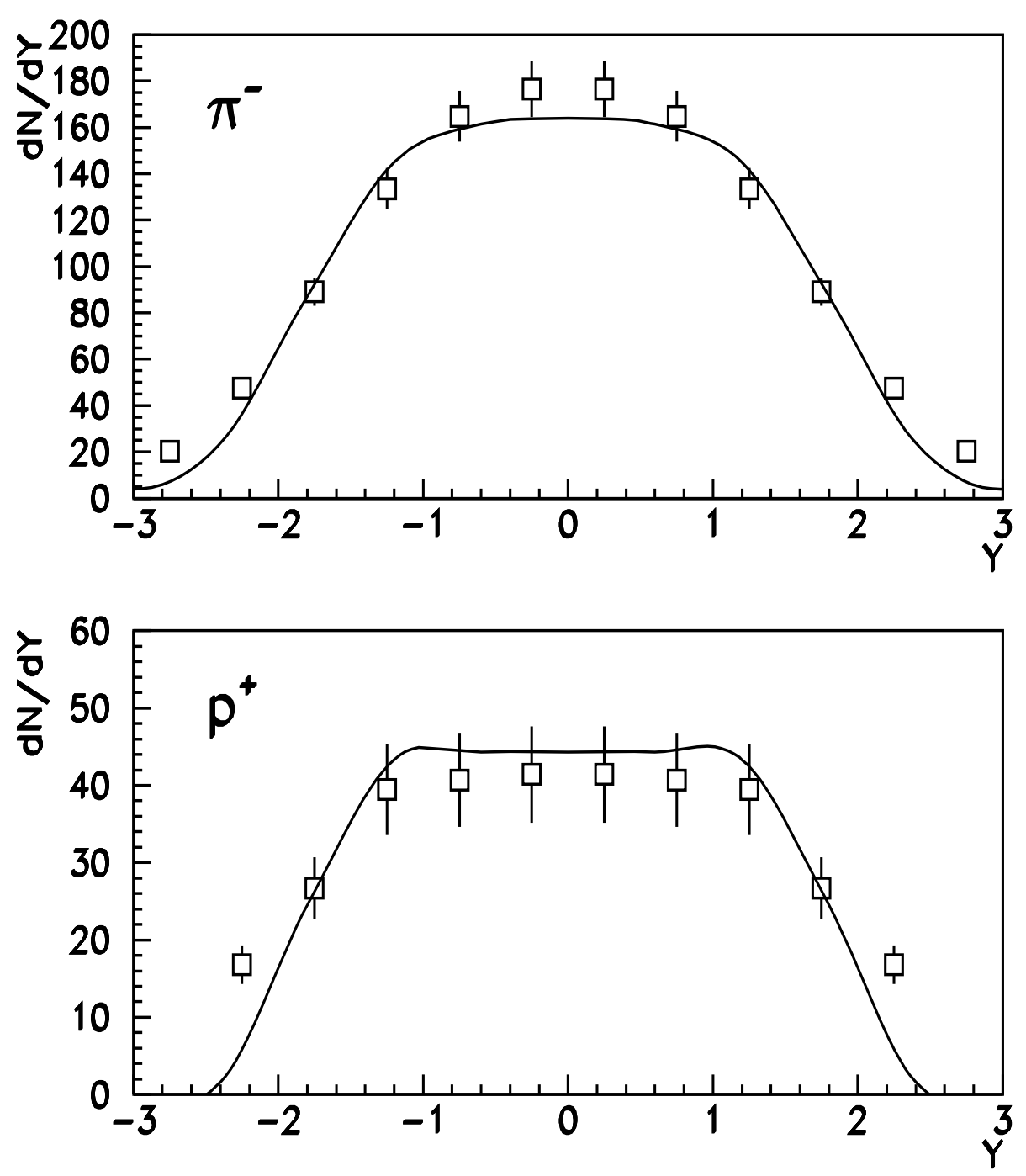

Fig. 1. Rapidity distributions of pions (top) and protons (bottom) from the extended ALCOR model for $160 \mathrm{AGeV} \mathrm{Pb}+\mathrm{Pb}$ central collisions. The open squares are the measured and the reflected data points for $h$ - particle (top) and '(+) - (-)' particle (bottom) spectra 18. The value of proton multiplicity at mid-rapidity is uncertain. In more recent publication 19] a small drop was given, but even the statistical errors are appreciable, yet. 

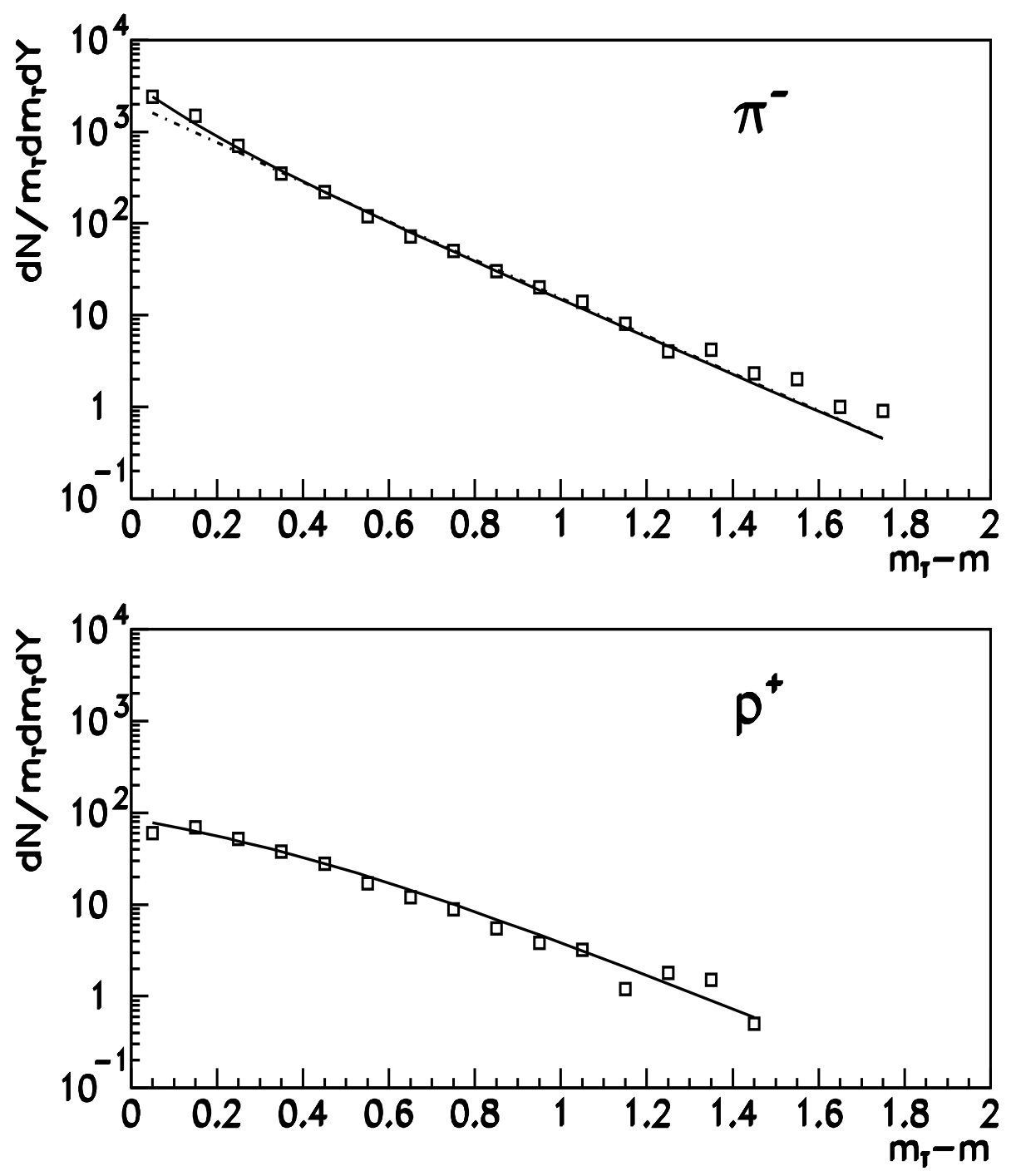

Fig. 2. Transverse mass, $m_{T}$, distribution of pions (top) and protons (bottom) calculated from ALCOR for $160 \mathrm{AGeV} \mathrm{Pb+Pb}$ collisions at mid-rapidity. The dotted line in the upper figure shows the result obtained with Boltzmann distribution. The open squares are the measured values for $h$ - particle (top) and '(+) - (-)' particle (bottom). 

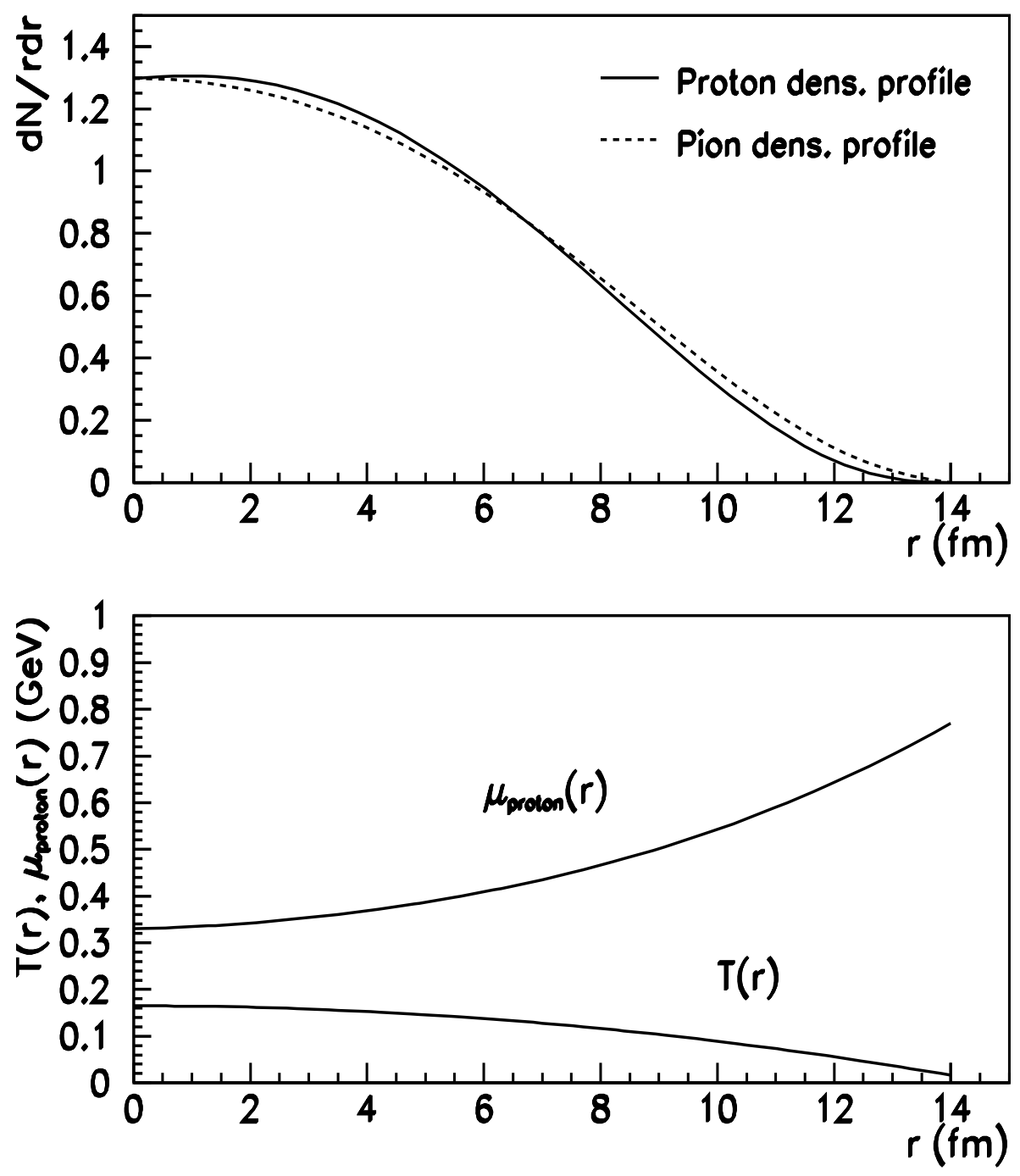

Fig. 3. The approximately similar radial density profiles for pions and protons (top) and the common temperature profile, $T(r)$ (bottom) together with the calculated chemical potential for protons, $\mu_{\text {proton }}$. The pion chemical potential was constant, $\mu_{\pi}=103 \mathrm{MeV}$. 
We would like to point out, that the description of the particle spectrum from the extended ALCOR model is based on a parameterization of the flow, density and temperature profiles of the hadronic phase in the time interval when the hot hadronic matter freezes out. This parameterization is qualitatively similar to the ones developed in refs. [20]-23], for the description of the single-particle spectra and the correlation function of the $\pi$ and $K$ mesons, as has been measured by the NA44 collaboration.

Both models confirm that the transverse flow profile, the local density profile and the transverse temperature profile [21]-23] may play a crucial role when describing the hadronic spectra.

\section{Conclusions}

In this paper we introduced the concept of transchemistry for the description of hadronization. The ALCOR model is a good approximation to the transchemistry description, if the hadronization is fast. The ratios of different hadron multiplicities obtained from ALCOR and from the thermal model - based on thermal and chemical equilibrium of hadron resonances - are much closer to each other, than one could expect. The rapidity and transverse mass distribution from ALCOR was compared to the experimental ones from the SPS $\mathrm{PB}+\mathrm{Pb}$ collisions. The agreement is good.

Further we have shown that the Bose statistics may play a role in the low $m_{t}$ enhancement in the transverse mass distribution.

These results support the claims that in high energy heavy ion collisions a semideconfined state of the matter is formed in $\mathrm{Pb}+\mathrm{PB}$ reactions at CERN SPS energies.

Since it seems that in the particle composition there are only minor, but systematic differences between ALCOR and thermal models, refined analysis tools are expected to play a critical role in the future data analysis: especially $\chi^{2}$ fits to the slope parameters and to the detailed invariant momentum distribution of various kinds of detected particles may be necessary to clearly distinguish among the proposed models.

Note also that the total volume and the inner dynamics characterized by the local temperature, flow and chemical potential distributions are very different in ALCOR and in the models assuming global thermal equilibrium. The HBT radius parameters are known to be very sensitive to the detailed structure of the flow, temperature and chemical potential profiles. Thus we expect that their evaluation using parameters fitted to the spectrum can possibly clearly distinguish between ALCOR and simple thermal models. Further work is necessary to evaluate this parameters from the models. 


\section{Acknowledgment}

Discussions with M. Gazdzicki, B. Müller and R. Stock are acknowledged. This work was supported by the National Scientific Research Fund (Hungary), OTKA No.T016206 and F4019 as well as by the U.S. - Hungarian Science and Technology Joint Fund, No. 378/93.

\section{References}

[1] I. Montvay, J. Zimányi, Nucl. Phys. A316 (1979) 490.

[2] T.S. Biró, J. Zimányi, Phys. Lett. B113 (1982) 6.

[3] T.S. Biró, P. Lévai, J. Zimányi, Phys. Lett. B347 (1995) 6.

[4] T.S. Biró, P. Lévai, J. Zimányi, Strangeness'95 Conference, 1995 Tucson, (AIP, Ed. J. Rafelski), AIP Conference Proceedings 340 (1995) 405.

[5] P. Csizmadia, P. Lévai, J. Zimányi, in preparation.

[6] P. Rehberg, S.P. Klevansky, J. Hüfner, Phys. Rev. C53 (1996) 410.

[7] D.U. Jungnickel, C. Wetterich, Phys. Rev. D53 (1996) 5142.

[8] T. Csörgö, L.P. Csernai, Phys. Lett. B333 (1994) 494.

[9] T.S. Biró, J. Zimányi, Nucl. Phys. A395 (1983) 525.

[10] P. Koch, B. Müller, J. Rafelski, Phys. Rep. 142 (1986) 167.

[11] J.D. Bjorken, Phys. Rev. D27 (1982) 140.

[12] e.g. L.I. Schiff, Quantum Mechanics,Chapt. 34, McGraw-Hill, New York, 1955

[13] J. Bächler et.al., NA35 Coll., Phys. Rev. Lett. 72 (1994) 1419.

[14] T.S. Biró, J. Knoll, H.B. Nielsen, Nucl. Phys. B245 (1984) 449.

[15] F. Cooper, G. Frye, Phys. Rev. D10 186.

[16] J. Zimányi, G. Fái, B. Jakobson, Phys. Rev. Lett. 43 (1979) 1705.

[17] P. Lévai and J. Zimányi, hep-ph/9609299, to appear in the Proceedings of the Workshop on QCD, 3-8 June 1996, AUP Paris, France.

[18] P. Seyboth, Proceedings of the XXV. International Symposium on Multiparticle Dynamics, Stara Lesna, 1995, World Scientific, Ed. by L. Sandor et al.

[19] See the recent results of NA49 Collaboration in the Proceedings of QM'96 to be published in Nucl. Phys. A.

[20] T. Csörgő and B. Lörstad, Nucl. Phys. A590 (1995) 465c.

[21] T. Csörgő and B. Lörstad, hep-ph/9509213, Phys. Rev. C (1996) (to be published), and references therein.

[22] T. Csörgö, P. Lévai and B. Lörstad, hep-ph/9603379, Acta Physica Slovaca 46 (1996) 585.

[23] T. Csörgö and B. Lörstad, Heavy Ion Physics 4 (1996) 213. 